\title{
ARTIFICIAL NEURAL NETWORKS BASED SLEEP MOTION RECOGNITION USING NIGHT VISION CAMERAS
}

\author{
Chung-Hsien Kuo, Fang-Chung Yang, Ming-Yuan Tsai, Ming-Yih Lee \\ Department of Mechanical Engineering, Chang Gung University, Tao-Yuan, Taiwan
}

\begin{abstract}
The body movement is one of the most important factors to evaluate the sleep quality. In
general, the sleep motion is hardly investigated, and it must take a long time to observe the motion of
the patient in terms of a pre-recoded video storage media with high speed playing. This paper
proposes an image-based solution to recognize the sleep motions. We use the contact free and IR-
based night vision camera to capture the video frames during the sleep of the patient. The video
frames are used to recognize the body positions and the body directions such as the "body up". "body
down". "body right". and "body left". In addition to the image processing, the proposed artificial
neural network (ANN) sleep motion recognition solution is composed of two neural networks. These
two neural networks are organized as in a cascade configuration. The first ANN model is used to
identify the body position features from the images: and the follower ANN model is constructed based
on the features that are identified by the first ANN model to recognize the body direction. Finally, the
implementations and the practical results of this work are all illustrated in this paper.
Biomed Eng Appl Basis Comm, 2004 (April); 16 : 79-86.
Keywords: artificial neural network, sleep motion recognition, machine vision, sleep quality
evaluation
\end{abstract}
\section{INTRODUCTION}

There are about 27 percent people who have insomnia problems, according to the surveys by the World Health Organization (WHO) on 25916 people in 14 countries and 15 centers [13]. Disease, irregular sleep, shift duty including overnight shift, pressure, and serious impact on real life will cause the reduction in the sleep quality. Sleep problem affects individuals of any age and occupation, and it also affects the growth of the society and economy. The sleep quality evaluations include the measurements of the sleep

Received: Jan 5, 2004; Accepted: Feb 6, 2004

Correspondence: Ming-Yih Lee, Professor

Department of Mechanical Engineering,

Chang Gung University 259, Wen-Hwa IsI Road,

Kwei-Shan, Tao-Yuan. Taiwan, 333

E-mail: leemiy@mail.cgu.edu.tw motion, body temperature, breath period and stertor.

In general, the sleep motion is hardly investigated. and it must take a long time to observe the motion of the patient in terms of a pre-recoded video storage media with high speed playing. In recent years, severa! literatures developed contact-based sensors $[2,6,8]$ to acquire the sleep motion of the patient. In 1992, Tamura et al. [8] proposed an optical fiber pressure sensor based solution to monitor the body movement during sleep in the bed. In this work, three optical sensors are installed in the bed. The body movement can be recognized according to the changes in the signal transitions.

Harada et al. [6] developed the pressure sensor sheet that is placed in the pillow. In this work, the pressure sensor sheet can detect the pressure distributions of the patient's head. The dynamics of the sleep motion can be recognizcd in terms of the changes in the pressure distributions. On the other hand, Cheng 
[2] proposed a strain gauge based solution to detect the sleep motions in 2002 . In this thesis, the strain gauges are mounted on a flexible steel bar. The steel bar is installed in the bed. The body movement will cause the deformation of both the flexible steel bar and the mounted strain gauges. Consequently, the measures of the deformation changes of the strain gauges are used to recognize the movements of the body.

Based on the previous discussions, we found that the contact-based sensors were widely used for the detections of the body motions during the sleep. However, the contact-based sensors are disadvantageous to the data acquisition and the sleep quality. The disadvantages are:

1. The contact-based sensors are installed in either the pillow or the bed, and they may affect the sleep quality. In this manner, the collected data is not objective.

2. Because the body curve is not a straight line, the sensors cannot completely detect the sleep motion, that is, some portions of the body may not contact the pressure sensors.

3. The steel bar may be fatigued after many tests, and such a fatigue increases the difficulties of calibration.

4. In addition to the body, there are many objects that are put in the bed. Such signal noises are hardly removed, and they will affect the detections of the body movements.

5. Finally, the pressure sensors may be improperly activated due to the collision of the hands or lags when the patient does not turn his body.

Due to the disadvantages of the contact-based pressure sensor, this paper proposed an image-based sleep motion recognition system using the artificial neural network (ANN) $[1,3]$. The neural network is an algorithm based software/ firmware component, which is constructed to emulate the learning, interpretation, etc. like the human brain. Therefore the neural network is capable of learning from training patterns, accumulating knowledge, applying the knowledge to other conditions, and finally making decision and conclusions.

Based on the proven neural network learning capability, several literatures used the neural network to recognize the human's motion. Guo et al. [5] used the stick figure model to present the human structure form the sequence of silhouette frames. The proposed methodology was constructed as two stages. The first stage retrieved and recorded the stick figure model from to represent the human silhouette in each frame. In the second stage, the time sequence of the stick figure parameter is transformed into the Fourier domain, and the first four Fourier component acted as the input nodes of the back-propagation (BP) neural network model to classify the stick figure model into the walking, running, and other motions.

In addition, Venkatesh Babu et al. [9] presented the compressed domain human motion recognition using motion history information. In this work, the method for constructing coarse motion history image (MHI) and motion flow image (MFI) from compressed MPEG video with minimal decoding was proposed. The MHI features were extracted based on the projection profiles and centroid; the MFI features were extracted based on affine motion model, projected 1-D feature, and 2-D polar feature. The extracted features were used to train the neural network to recognize a set of seven human actions such as "walk", "run", "jump", "body-up", "body-down", etc. based on the Bayes classifier.

In this paper, the contact free and IR-based night vision camera is used to recognize the sleep motion so that the sleep quality can not be affected during acquisitions. The proposed solution is composed of two neural networks. These two neural networks are organized as in a cascade configuration. The first ANN model is used to identify the body position features from the images; and the follower ANN model is constructed based on the features that are identified by the first ANN model to recognize the body direction. Finally, the implementations and the practical results of this work are all illustrated in the following sections.

\section{METHODS}

\subsection{System Development Architecture}

\subsubsection{Hardware setup and configuration}

This paper proposes an image-based solution to recognize the body movement during sleep. In order to provide an accurate data acquisition methodology that does not affect the sleep quality during detection, this work uses an IR-based night vision camera to capture the video frames. The night vision CCD (Charged Coupled Device) camera is manufactured by TA-FU Electronic Technology Co., Ltd. with the type of TF20M/IRB6 [11]. The video frames are captured using the Matrox Meteor-II [10] analog monochrome/ color video acquisition card. The night vision camera is located at the overhead space of the bed. The view of the camera can cover all the bed, as shown in Fig. 1.

\subsubsection{Image recognition architecture}

In this section, the image recognition architecture is proposed. A multi-layer feed-forward backpropagation (BP) neural network [3] is used to recognize the sleep motions. The neural network is a network of composing the neurons. The neurons act as the nonlinear computing elements that organize to 
form a computational model. The interconnected neurons can train or learn the underlying associations and casual relationships in terms of the training patterns. Therefore, the ANN models are appropriate to recognize the sleep motions.

The system development procedures are indicated as shown in Fig. 2. The images are grabbed using the Meteor-II image acquisition card, and these images are filtered and pre-processed initially so that they can be easily characterized. In order to meet the input characteristics of the neural network models, the edge detection, binarization, gray level statistics, images row/ column profile projections, and so forth $[4,10]$ are all done for the further ANN models' trainings and recognitions. Consequently, the body movements are defined over the body positions and the body directions such as "body up", "body down", "body right", and "body left".

On the other hand, the body movement recognition procedures can be categorized into the following stages.

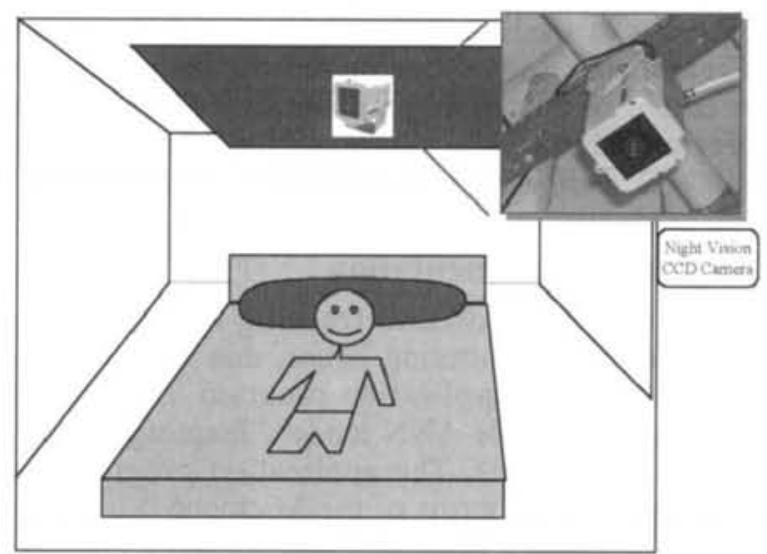

Fig. 1 System setup and night vision camera.

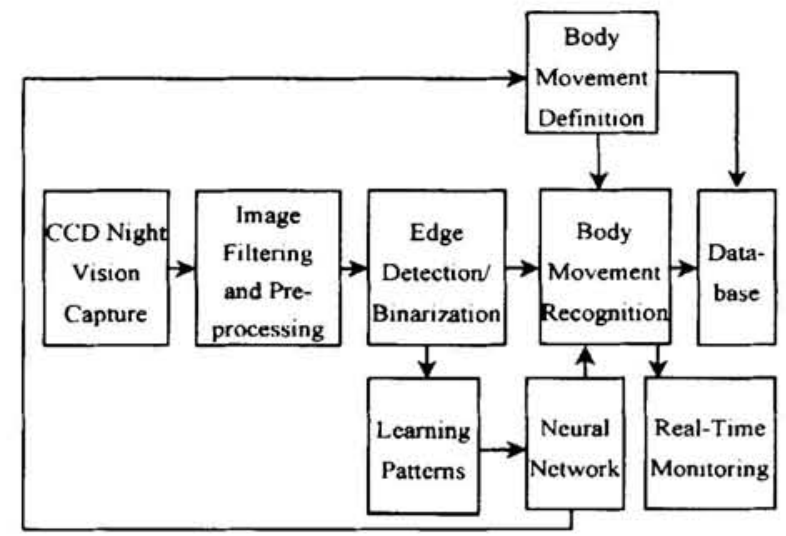

Fig. 2 System development procedures.
1. Acquire the image patterns of different body movements that are defined over the body positions and directions.

2. Define the input and output characteristics from the images.

3. Apply the neural network model to train the images patterns until the convergence of the weights.

4. Use the recall function and input characteristics to recognize the body positions and directions. The results are dynamically shown in the monitoring program, and they are also stored in the database.

5. Validate the recognition results in terms of investigating the video frames.

\subsection{Applying the Artificial Neural Networks}

In this work, the proposed sleep motion recognition solution is composed of two layers' neural networks. These two neural networks are organized as in a cascade configuration. The first ANN model is used to identify the body position features from the images, as indicated in the subsection 2.1.1; and the follower ANN model is constructed based on the features that are identified by the first ANN model to recognize the body direction, as indicated in the subsection 2.1.2. Based on the proposed learning architecture, the body positions and directions can be recognized.

\subsubsection{Body position recognition ANN model}

Since the patient is not always lying in the center of the bed, the position transitions of lying down are required to be measured. In addition, the positions of lying down can also be used to identify the image regions for the further body direction recognition. In this work, the body position is defined as an image region that is measured from the patient's shoulder to his crotch, as shown in Fig. 3.

The proposed ANN model uses the feed-forward backpropagation (BP) neural network with two hidden layers, as shown in Fig. 4. In this ANN model, the input nodes are defined as the row and column profile projections $[4,10]$ of the original images. The original image is a $640(\mathrm{H}) \times 480(\mathrm{~V})$ pixels resolution. The gray level of each pixel is defined as $G(x, y)$ where $x=$ 1 to $640 ; y=1$ to 480 . Note that each profile projection is defined as the normalized ( 0 to 1$)$ average gray level valuc in a rectangle area with $s$ (in this model, $s=20$ ) pixels width or height. The 56 input nodes $\left(\mathrm{IN}_{1}\right.$ to $\left.\mathrm{IN}_{56}\right)$ are defined as in (1) and (2):

Row profile image projections $(i=1$ to 24$)$ :

$$
I N_{i}=\sum_{j=1}^{s} \sum_{x=1}^{640}(G(x, y+s \times(i-1)) / s \times H \times 255
$$


Column profile image projections $(\mathrm{i}=25$ to 56 ):

$$
I N_{i}=\sum_{x=1}^{s} \sum_{y=1}^{480} G(x+s \times(i-25), y) / s \times V \times 255
$$

The output of this ANN model is defined as the body position which is represented as the image area of the position where the patient is lying down, as indicated in Fig. 3. In the proposed ANN model, the four output nodes are defined as the $\mathrm{x}$ coordinates and $y$ coordinates of the left upper [that is (X_LU, Y_LU)] and right down [that is (X_RD, Y_RD)] corners of the image area of the body position, respectively.

\subsubsection{Body direction recognition ANN model}

In the first ANN model, the body position that is represented as an image area is recognized. The recognized image areas can also be used to identify the possible head image areas of the patients from the captured images. In this portion, we also use the profile image projections of the head image area to recognize the face directions such as "up", "down", "left", and "down". The body direction is defined the same as the face direction in this paper. The proposed body direction ANN model also uses the feed-forward backpropagation neural network with two hidden layers, as similarly shown in Fig. 4 except the numbers of input, output and hidden neurons.

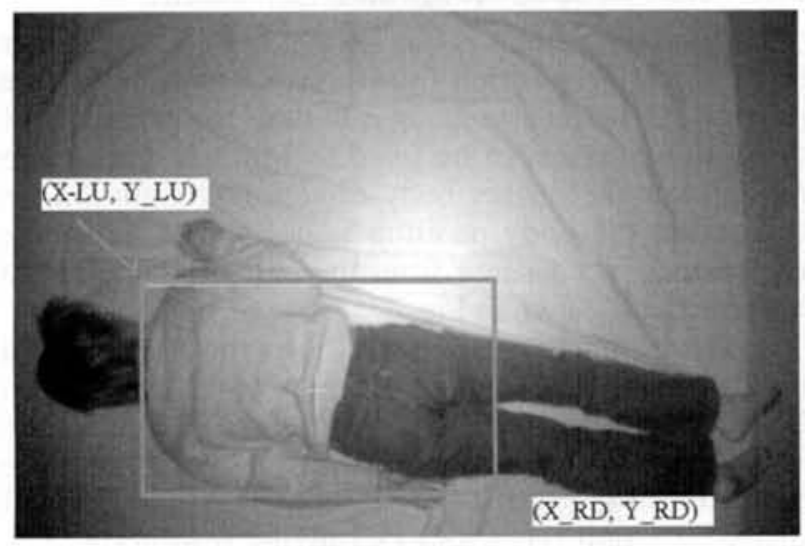

Fig. 3 Body position definition.

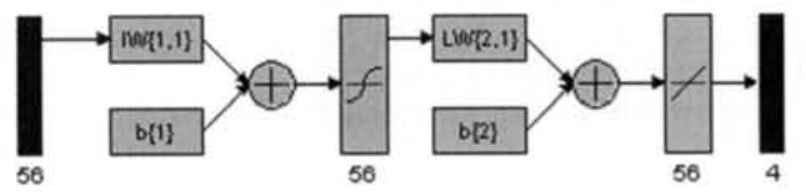

In this ANN model, the input nodes are defined as the row profile projections of the possible head image area. Since the vertical distance between the bed and the camera is invariant, the size of head image area is almost independent of the position of the patient. In this work, the possible head image area is defined as a $100(\mathrm{H}) \times 200(\mathrm{~V})$ pixels area. This image area is varying according to the body position, as recognized in the first ANN model. Note that each profile projection is also defined as the normalized (0 to 1$)$ average gray level value in a rectangle area with height $s$ (in this model, $s=4$ ) pixels width. The 50 input nodes $\left(I_{1}\right.$ to $\left.I_{50}\right)$ are defined as in (3):

Row profile image projections ( $\mathrm{i}=1$ to 50 ):

$$
\begin{aligned}
I N_{i}= & \sum_{y=0}^{s-1} \sum_{x=0}^{99} G(H x+x, H y+y+s \times(i-1)) / \\
& s \times H \times 255
\end{aligned}
$$

Note that the $\mathrm{Hx}$ and $\mathrm{Hy}$ are $\mathrm{x}$ - and $\mathrm{y}$-coordinates of the left upper corner of the head image area. They are limited to $1 \leqq \mathrm{H} x \leqq 320$ and $1 \leqq \mathrm{H} y \leqq 281$ to ensure the reasonable and proper operation of the program. The output of this ANN model is defined as the body direction where " 0 " indicates "up"; " 1 " indicates "right"; "2" indicates "left"; and "3" indicates "down".

\subsection{System Implementation}

Based on the offline training and the online recognition and monitoring issues, this work develops a windows-based application program to implement the proposed cascade ANN models' learning functions and recall functions. The application program (i.e., sp.exe) is coded in terms of the Microsoft Visual C++ [7] and the Matrox Imaging Library (MIL) [10]. MIL is a software development toolkit for the machine vision, medical imaging and image analysis. This application program is constructed in an objectoriented manner. The classes of the image processing [4] and feed forward BP neural network are constructed.

The development architecture of the application program is shown in Fig. 5. In this figure, four major modules were constructed. The file server is used to store the captured training image patterns. The access database is used to record the features of training image patterns, ANN BP model structure weightings, and sleep motion recognition results. The open database connectivity (ODBC) [12] is responsible for connecting the Access database and the application program.

Fig. 4 Body position ANN model structure. 
On the other hand, the Mill image library is used to capture the video frames from the IR-based night vision camera for the training image patterns and inspection video frames. In addition, the image processing and analysis modules are constructed to manipulate the captured images such as variable video size zooms, offset adjusting, Sobel edge detection [4, $10]$ and binarization. Meanwhile, the image feature extraction module for the ANN model inputs, as described in previous subsection are also included.

The ANN model is the kernel of this work. The ANN BP software component is developed in this work. The model structure of the numbers of input nodes, output node and hidden layers can be flexibly assigned. The input nodes and training patterns can be imported to the ANN software module. The training and echo engines are constructed for the learning and recognition purposes. The learn capability can also be destined. Simultaneously, the learning efficiency and convergence are addressed. The user interface of the proposed ANN BP software module is shown in Fig. 6 .

Finally, the graphical user interface (GUI) is shown in Fig. 7. The original image is shown in the left upper comer of application GUI. The functions can be operated and investigated in terms of the GUI components, and they are classified into 5 categories. They are further illustrated as follows:

1. Image information: it includes the image file path and name during the training pattern recording, offline line training, or offline batch recognition stages, and the live video frame ID.

2. Image processing and analysis: it includes the operations within the image acquisition, variable size zooms, offset adjusting, Sobel edge detection $[4,10]$ and binarization.

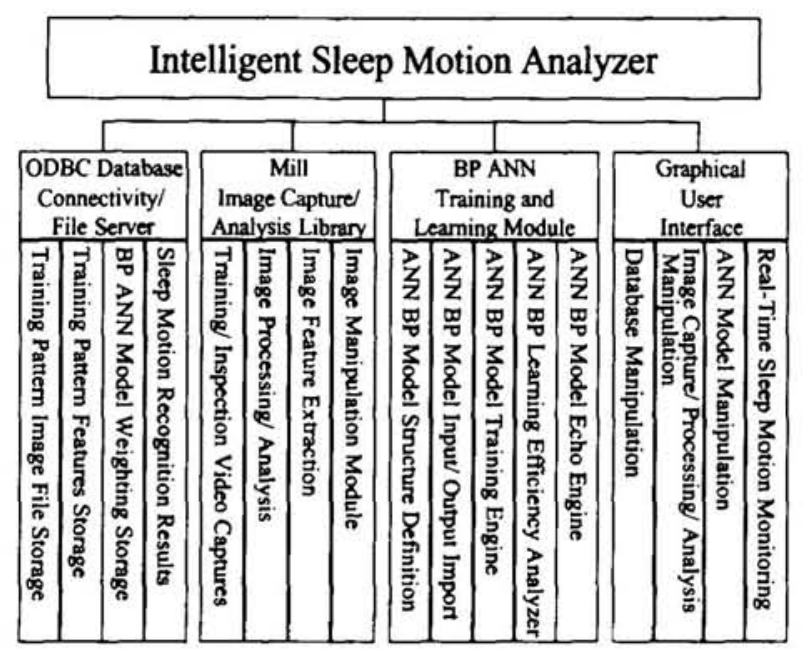

Fig. 5 Application program development architecture.

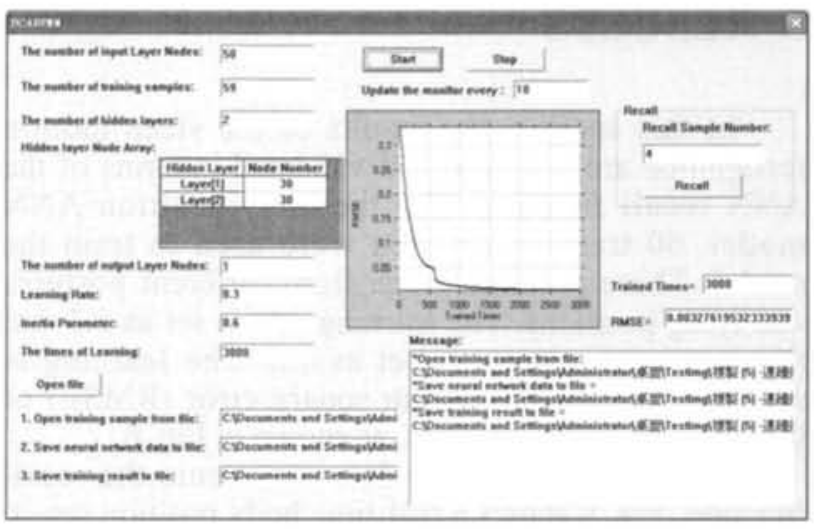

Fig. 6 ANN BP software module.

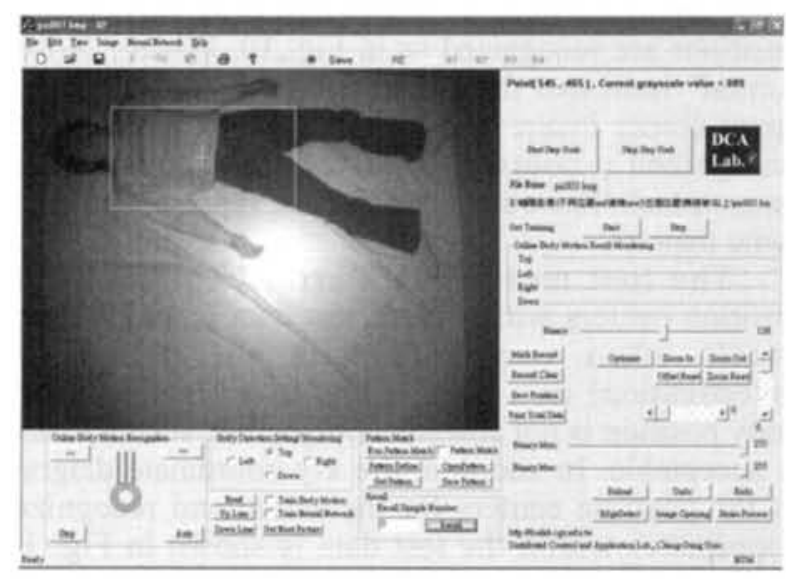

Fig. 7 User interface of the application program.

Especially, this work also includes the pattern matching functions $[4,10]$ to analyze the images. Consequently, the input nodes for the ANN models, as shown in equations of (1) to (3), are all generated here.

3. Training patterns recording: this function aims to open the image patterns, and then mark the desired body position image area and the body direction. Note that these records are stored in the database as the output nodes of the training patterns.

4. Neural network operating: it can execute the learning and recall functions of the ANN models.

5. Monitoring: the recall results are dynamically represented as the body direction indicator, body position marker, and historical body direction chart. 


\section{RESULTS}

In this section, the results of the sleep motion recognition are analyzed and validated in terms of the ANN recall function. For the body position ANN model, 60 training patterns were used to train the model. These patterns range from different postures and lying positions. The learning rate is set as 0.3 and the inertia parameter is set as 0.6 . The learning is converged at the root mean square error (RMSE) of 0.0068 after 2000 iterations, as shown in Fig. 8 .

We also used 29 test data to execute the recall function. Fig. 9 shows a real-time body position result, which is recognized in terms of the recall function. Consequently, four output nodes that indicate the $x$ coordinates and y coordinates of the left upper and right down corners of the image area of the body positions are recognized as in Fig. 10. Where "X-LUActual" indicates the actual $\mathrm{x}$ coordinate of the left upper corner image pixel; "Y-RD-ANN" indicates the recognized y coordinate of the right down corner image pixel. The others can also be represented in the same manner.

The root mean square error (RMSE) of the position centers within these test data is 10.79 image pixels when compared with the actual (by investigation) data. Due to the investigation of the body position is not absolutely objective, such a RMSE is acceptable. In addition, the $x-y$ coordinate diagram of the position centers for the actual and recognized coordinates within the test data is shown in Fig. 11. The body position of "body up", "body down", "body left", and "body right" can all be recognized, as shown in Fig. 12.

Meanwhile, the body direction ANN model is also analyzed. The proposed ANN model is trained under

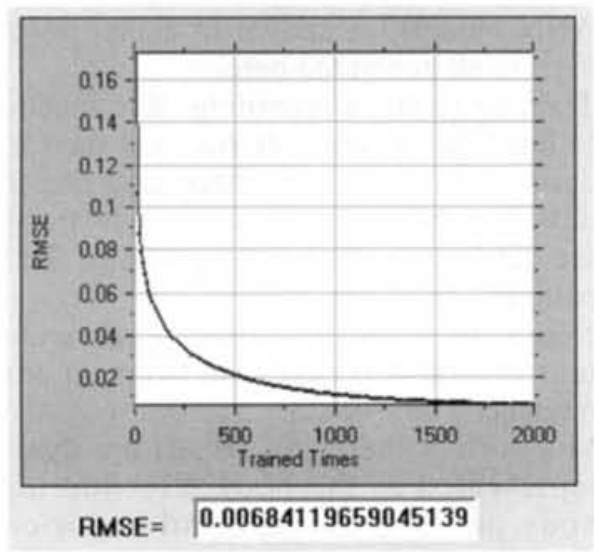

Fig. 8 Learning convergence of body position model.
69 training patterns. These patterns cover from the different postures and lying positions. The learning rate is set as 0.3 and the inertia parameter is set as 0.6 . The learning is converged at the root mean square error (RMSE) of 0.0066 after 2000 iterations, as shown in Fig. 13. Fig. 14 shows the comparisons of the actual direction, ANN recalled value (in a float value) and recognized direction (in a round integer value) for the 28 test data. There is a recognition error (test sequence ID: 17) among these test results.

On the other hand, we also discuss the case of using the bedquilt. Since this paper does not use the ANN model to learn the case of when the patient is in bedquilt, the pattern matching technique is used to discuss the using bedquilt condition. Four patterns of the "face up", "face right", "face left", and "face down" for a specified patient are all defined categorically, and the system can recognize the head image area and the body direction as well. Fig. 15 shows the dynamic monitoring the body direction using the pattern matching techniques $[4,10]$ when the patient is in bedquilt.

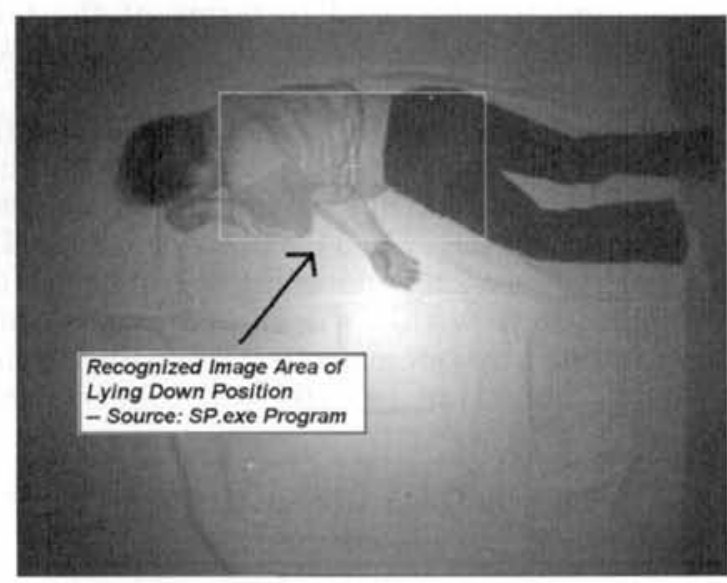

Fig. 9 Body position recognition.

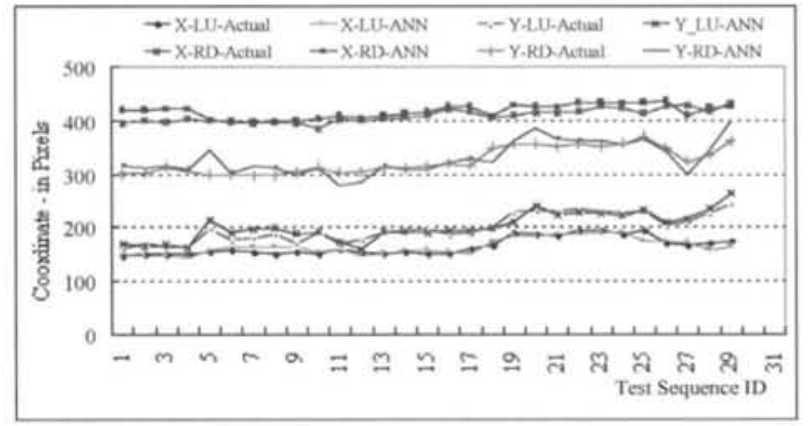

Fig. 10 Actual and recognized body position output nodes. 


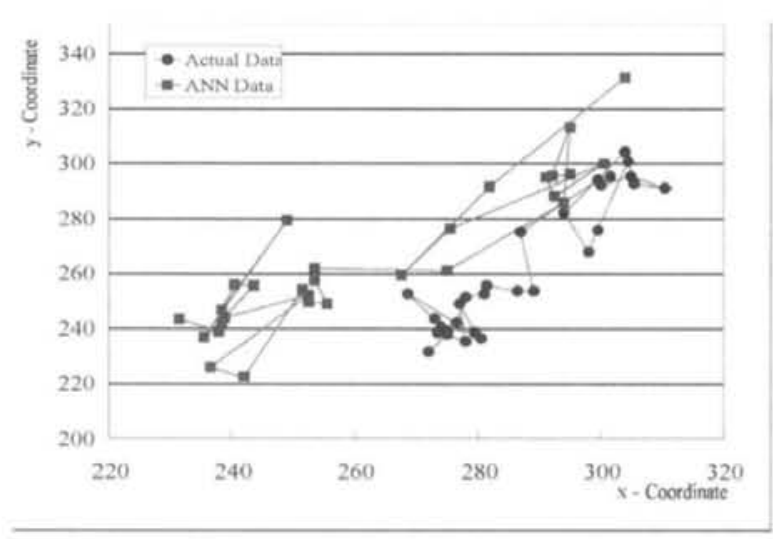

Fig. 11 Actual and recognized body position - x-y plots.
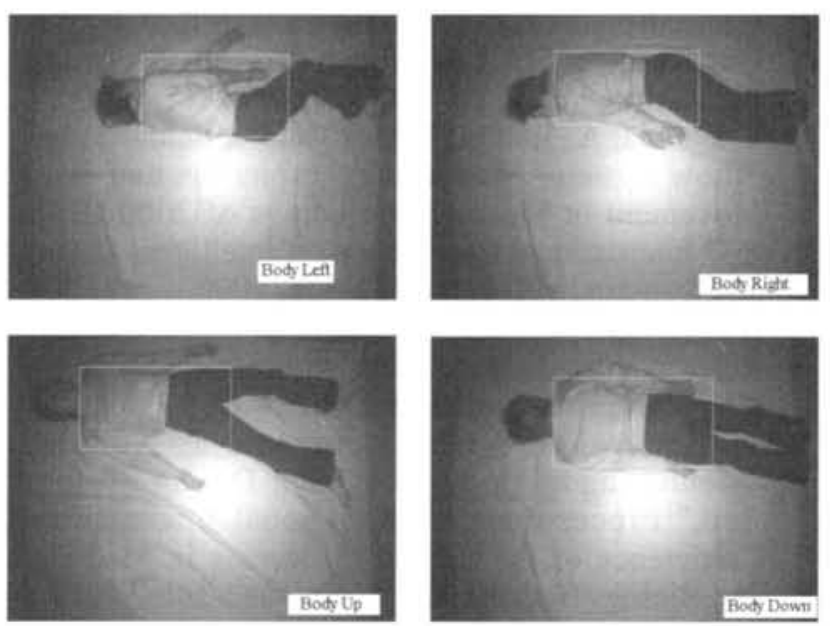

Fig. 12 Body position of recognition of different postures.

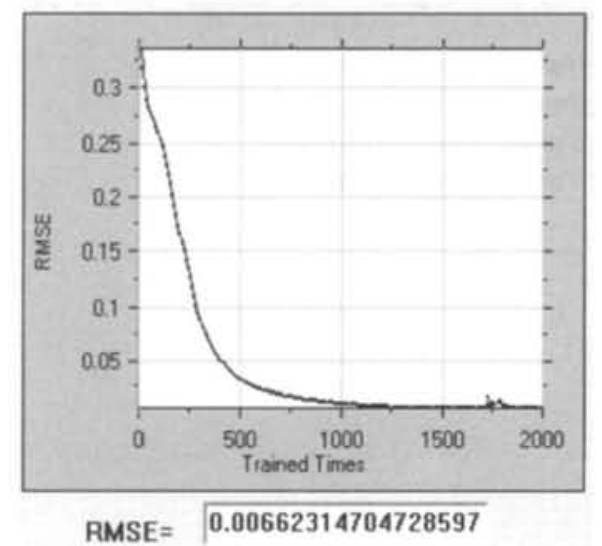

Fig. 13 Learning convergence of direction model.

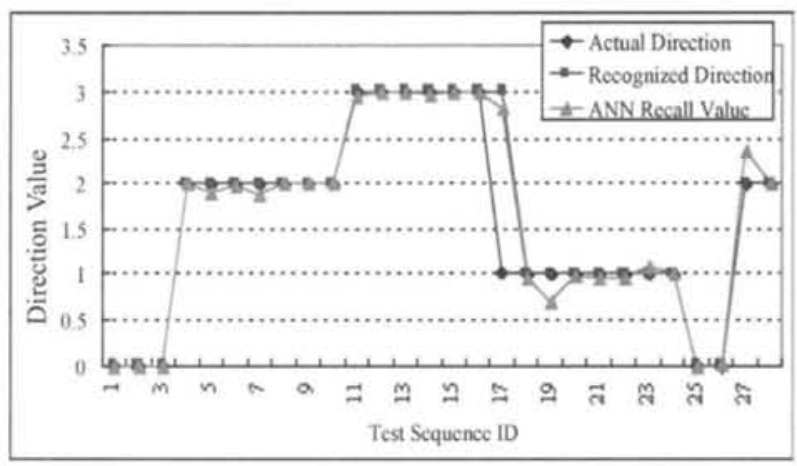

Fig. 14 Comparisons of the actual directions, ANN recalled values and recognized directions.

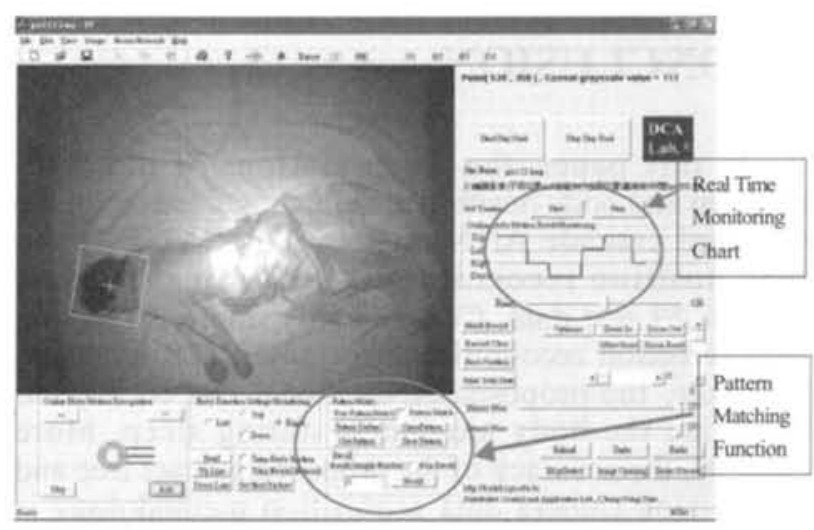

Fig. 15 Dynamically monitor the body direction when the patient is in bedquilt.

\section{DISCUSSION}

This paper proposed a contact free IR-based night vision CCD camera solution to inspect the sleep motions. Such a solution resolves the problems of contact based sensor solution that may affect the accuracy and objectivity of the inspection. Especially, the discomfort of inserting the contact sensors really affects the sleep quality during inspection. On the other hand, the night vision based inspection consists with the regular sleep situation. Meanwhile, the noises resulting from nonsensical collisions of the pressure sensors can be removed. Therefore, the proposed sleep motion inspection methodology is comfort, objective, and convenient.

In addition, the intelligent based ANN training and learning system enhances the accuracy of sleep motion recognition. Although the current results show acceptable results according to the previous results, there are still several works are keeping proceeding. 
1. The measured sleep motion data is collected, and now, we are cooperating with the physicians to discuss the clinical applications. Several insomnia cases are under inspection.

2. Currently, the limitation of the proposed vision inspection system cannot accept the bedcover with complex colors. This can be simply solved by arranging the inspection environment. However, we are trying to develop new version of system that is adaptive to more flexible inspection environment.

3. The current system can be used for the patient with specific hairstyle. That is, different hairstyle must re-train the system again. The new ANN model structure can be proposed in the future to enhance the model's adaptivity.

\section{CONCLUSION}

In this paper, the sleep motions of the body direction and body position are all recognized using the proposed cascade ANN models. The RMSE of the body position recognition is 10.79 image pixels through 29 test data. Consequently, there exists one body direction recognition error among 28 test data. Therefore, the proposed ANN model can be used to recognize the body movement during sleep. More specially, the accuracy of the proposed contact free and night vision camera data acquisition methodology is acceptable, and this method does not affect the sleep quality during acquisition. However, this approach can be used to recognize the patient with similar hairstyle and clothes dressing currently. In the future, the ANN model will be redefined to recognize the patient with wide application variations such as hairstyle, clothes dressing, and using the bedquilt. Finally, the results of this work will be also redefined to meet the requirements of the clinical evaluation and diagnosis.

\section{ACKNOWLEDGEMENT}

This work was supported by the Medical Mechatronics Education Resource Center, Ministry of Education, Taiwan, R.O.C., under the 2002 Student Hand-On Competition Project.

\section{REFERENCES}

1. T. Chen, W.C. Lin, and C.T. Chen, "Artificial Neural Networks for 3-D Motion Analysis - Part II: Nonrigid Motion," IEEE Transactions on Neural Networks, Vol. 6, No. 6, pp. 1394-1401, 1995.

2. C.M. Cheng, "Design of a Sleep Monitoring and Evaluating System," Master Thesis, Graduate Institute of Mechanical Engineering, Yuan Ze University, Taiwan, 2002.

3. J.A. Freeman and D.M. Skapura, Neural Network Algorithms, Applications, and Programming Techniques, New York:Addison-Wesley, 1992.

4. R.C. Gonzalez and R.E. Woods, Digital Image Processing, 2nd Edition, London:Prentice-Hall, 2002.

5. Y. Guo, G. Xu, S. Tsuji, "Understanding Human Motion Patterns," Processing of 12th IAPR International Conference on Pattern Recognition Conference B: Computer Vision \& Image Processing, Vol. 2, pp. 325-329, 1994.

6. T. Harada, A. Sakata, T. Mori, and T. Sato, "Sensor Pillow System: Monitoring Respiration and Body Movement in Sleep," Proceedings of 2000 IEEE/ RSJ International Conference on Intelligent Robots and Systems, Vol. 1, pp. 351-356, 2000.

7. D.J. Kruglinski, Inside Visual C++ - The Standard Reference for Programming with Microsoft Visual C++ Version 4, Washington:Microsoft, 1996.

8. T. Tamura, A. Nishigaichi, and T. Nomura, "Monitoring of Body Movement During Sleep in Bed," Proceedings of the Annual International Conference of the IEEE Engineering in Medicine and Biology Society, Vol. 4, pp. 1483-1484, 1992.

9. R. Venkatesh Babu and K.R. Ramakrishnan, "Compressed Domain Human Motion Recognition Using Motion History Information," Processing of IEEE International Conference on Acoustics, Speech, and Signal Processing, Vol. 3, pp. 41-44, 2003.

10. http://www.matrox.com/

11. http://www.tafu-cetv.com.tw/

12. http://www.microsoft.com/

13. http://www.who.int/en/ 